\title{
Skin Carotenoid Response to a High-Carotenoid Juice in Children: A Randomized Clinical Trial
}

\author{
Sheryl S. Aguilar \\ Utah State University \\ Heidi J. Wengreen \\ Utah State University \\ Jeffrey P. Dew \\ Brigham Young University - Provo, jeff_dew@byu.edu
}

Follow this and additional works at: https://scholarsarchive.byu.edu/facpub

Part of the Other Social and Behavioral Sciences Commons

\section{Original Publication Citation}

Aguilar, S. S.*, Wengreen, H. J., \&Dew, J. P. (2015). Skin carotenoid response to a high-carotenoid juice in children: A randomized clinical trial. Journal of the Academy of Nutrition and Dietetics, $115,1771-1778$.

\section{BYU ScholarsArchive Citation}

Aguilar, Sheryl S.; Wengreen, Heidi J.; and Dew, Jeffrey P., "Skin Carotenoid Response to a High-Carotenoid Juice in Children: A Randomized Clinical Trial" (2015). Faculty Publications. 4532.

https://scholarsarchive.byu.edu/facpub/4532

This Peer-Reviewed Article is brought to you for free and open access by BYU ScholarsArchive. It has been accepted for inclusion in Faculty Publications by an authorized administrator of BYU ScholarsArchive. For more information, please contact ellen_amatangelo@byu.edu. 


\title{
Skin Carotenoid Response to a High-Carotenoid Juice in Children: A Randomized Clinical Trial
}

\author{
Sheryl S. Aguilar, MS, RD; Heidi J. Wengreen, PhD, RD; Jeffrey Dew, PhD
}

\author{
ARTICLE INFORMATION \\ Article history: \\ Submitted 23 December 2014 \\ Accepted 5 June 2015 \\ Available online 5 August 2015

Keywords:
Carotenoids
Children
Raman spectroscopy
Vegetables
ASA24

2212-2672/Copyright ( $\odot 2015$ by the Academy of Nutrition and Dietetics.

http://dx.doi.org/10.1016/j.jand.2015.06.011

\begin{abstract}
Background Previous studies have shown an increase in serum carotenoid status among children when fed carotenoids. This study looked at the effect and dose-response of a known amount of carotenoid consumption on change in skin carotenoid status among children.

Methods Participants were children aged 5 to 17 years from Cache County, UT ( $n=58$ ). Children were randomly assigned to one of three groups: high $(n=18)$ or low $(n=18)$ dose of a carotenoid-rich juice (2.75 $\mathrm{mg}$ carotenoids/30 mL juice), or placebo juice $(n=22)$. Children were asked to drink an assigned dose of the juice (30 to $120 \mathrm{~mL} /$ day) based on the weight of the child and group assignment, every day for 8 weeks. Skin carotenoids were measured every 2 weeks by resonance Raman spectroscopy. Participants were asked to maintain their usual diet throughout the study. Usual diet was assessed using three averaged 24-hour recalls; diet constancy was measured using food frequency questionnaires administered at baseline, Week 4, and Week 8. Repeated measures analysis of variance was used to assess the group differences in skin carotenoid status over time.

Results The high-dose and low-dose groups had mean \pm standard deviation increases in skin carotenoid status of $11,515 \pm 1,134$ and $10,009 \pm 1,439$ Raman intensity counts, respectively (both $P$ values $<0.001$, for change in means compared with baseline) at Week 8 , although they showed significant change from baseline by Week 2 . The placebo group's change of 985 Raman intensity counts was not statistically significant. The difference in change between the 2 experimental groups was not significant at Week 2, 4, 6, or 8 .

Conclusions Consumption of 30 to $120 \mathrm{~mL}$ ( 2.75 to $11 \mathrm{mg}$ carotenoids) of a carotenoidrich juice significantly increased skin carotenoid status over an 8-week period among children aged 5 to 17 years. The amount of carotenoids found in this amount of juice is equal to the amount found in approximately 23 to $92 \mathrm{~g}$ cooked carrots per day.

J Acad Nutr Diet. 2015;115:1771-1778.
\end{abstract}

$\mathrm{N}$ UMEROUS STUDIES HAVE DEMONSTRATED THAT fruits and vegetables $(\mathrm{F} / \mathrm{V})$ can be protective against oxidative damage ${ }^{1-3}$ and people who eat high amounts of $\mathrm{F} / \mathrm{V}$ have lower risk for mortality and many chronic diseases. ${ }^{4}$ Highly pigmented red, orange, yellow, and green $\mathrm{F} / \mathrm{V}$ are good sources of carotenoids as well as other antioxidants. Carotenoid status may be a biomarker of antioxidant status and $\mathrm{F} / \mathrm{V}$ intake. ${ }^{5,6}$

Carotenoids are fat-soluble pigments and when eaten are transported in the blood in lipoproteins and deposited in the lipid layer of the epidermis of human beings. Levels can be assessed by measuring the concentration of carotenoids in blood or skin. Previous studies in adults ${ }^{7}$ and children ${ }^{8}$ have identified strong correlations $(r=0.81$ and $r=0.79$, respectively; both $P$ values $<0.001$ ) between the amount of

To take the Continuing Professional Education quiz for this article, log in to www.eatrightPRO.org, go to the My Account section of the My Academy Toolbar, click the "Access Quiz" link, click "Journal Article Quiz" on the next page, and then click the "Additional Journal CPE quizzes" button to view a list of available quizzes. carotenoids measured in the blood using high-performance liquid chromatography and the amount measured in the skin, using resonance Raman spectroscopy (RRS).

Primary carotenoids detected in the skin are lycopene, beta carotene, alpha carotene, beta cryptoxanthin, lutein, zeaxanthin, phytoene, and phytofluene, with lycopene present in the highest amounts. ${ }^{9}$ Carotenoid levels in the diet have been shown to be significantly correlated with skin carotenoid status. ${ }^{8,10-13}$

Studies have found that providing beta carotene supplements, ${ }^{14,15}$ high-carotenoid fruit, ${ }^{15}$ or high-carotenoid vegetables ${ }^{14,16,17}$ increase plasma beta carotene levels quickly in children. Jahns and colleagues ${ }^{18}$ found that skin carotenoid status measured by RRS closely followed changes in plasma carotenoid values in adults as total dietary carotenoid intake increased and decreased. ${ }^{18}$

The purpose of this study was to examine changes in skin carotenoid status in children, measured by RRS over an 8week period of time when a known dose of carotenoids was consumed in the form of juice with standardized carotenoid content. 


\section{METHODS}

\section{Participants}

A total of 67 healthy Cache County, UT, schoolchildren aged 5 to 17 years were recruited through local elementary and secondary schools during December 2012. The Institutional Review Board at Utah State University reviewed and approved the research protocol. A letter of information was sent via e-mail to parents of a local elementary school. Older children were recruited by word of mouth and from siblings of elementary schoolchildren. Researchers recruited a representative sample of approximately $8 \%$ of study children from each grade kindergarten through $12,47 \%$ of whom were boys. The race/ethnicity of the participant population reflected the race/ethnicity of the local school-age population in Cache County, UT (80\% white, $17 \%$ Hispanic, and $<3 \%$ Asian and Pacific Islander). Parents of children completed a qualifying online survey. In the case that the child met the study inclusion/exclusion criteria, the parents were mailed/emailed and asked to complete a Center for Human Nutrition Studies health history questionnaire that includes past and present medical history, past and current medication use, and nutritional supplement use. Children were excluded if they had a health history or habits that were known to affect carotenoid levels. ${ }^{19}$ These exclusions included major illness during the 2 weeks before the study began, use of carotenoid supplements (multivitamins were allowed), use of topical self-tanning lotion, chronic disease such as asthma or type 1 diabetes, and sun exposure for $>2$ hours/day without use of sunscreen. Three children were excluded from the study due to a reported history of asthma and one due to rheumatoid arthritis.

Researchers obtained parent consent and child assent from participants in person. Participants were asked to maintain their normal lifestyle, including activity, multivitamin use, dietary pattern, and physical activity habits for the duration of the study. Participants received $\$ 10$ at clinic visits 2, 4, 6, and 8 and a $\$ 20$ bonus for completing the study, for a total of $\$ 60$.

\section{Protocol}

Qualifying children had a BioPhotonic Scanner (NuSkin, LLC) score, indicating skin carotenoid status, between 11,000 and 32,000 Raman intensity counts. The range was limited to eliminate subjects with abnormally high or low counts and to stay within 2 standard deviations from the average score for school-aged children of approximately 22,000 Raman intensity counts (information provided by the manufacturer, NuSkin, LLC). Participants completed nine clinic visits scheduled 7 days apart at the Center for Human Nutrition Studies clinic at Utah State University. During the baseline clinic visit, height was measured using a Seca 223 digital stadiometer and weight was measured using a Detecto $758 \mathrm{C}$ digital scale. Height and weight $\left(\mathrm{kg} / \mathrm{m}^{2}\right)$ were used to calculate body mass index (BMI). BMI was categorized based on age and sex percentiles.

Participants were assigned sequentially into a randomgenerated matrix by the study coordinator. They were assigned to one of the three treatment groups (see Figure 1): Carotenoid-Rich Active-High (AH) $(\mathrm{n}=18)$, Carotenoid-Rich Active-Low (AL) $(n=18)$, or Placebo $(P)(n=22)$. Participants then received either a high dose of juice $(30 \mathrm{~mL}$ twice per day if participant's weight was $<39 \mathrm{~kg}$ or $60 \mathrm{~mL}$ twice per day if $\geq 39 \mathrm{~kg}$ ) or a low dose of juice ( $15 \mathrm{~mL}$ twice per day if participants weight was $<39 \mathrm{~kg}$ or $30 \mathrm{~mL}$ twice per day if $\geq 39$ $\mathrm{kg}$ ), according to group assignment. Participants were asked to drink their prescribed amount of juice with a meal or snack for 8 weeks. The carotenoid-rich juice is commercially available under the name g3 Juice (NuSkin, LLC) and contained $2.75 \mathrm{mg}$ carotenoids per $30 \mathrm{~mL}$. The lot of juice that was used in the study was validated by a clinical laboratory (NuSkin Enterprises). Bottles were labeled with the same three-digit number on all of the placebo juice and a different number on the carotenoid-rich juice. Juice was measured and distributed by trained research assistants during weekly clinic visits. Research assistants were informed which bottle number to give to participants but did not know what that number represented. To check compliance, participants were asked to bring all juice containers (glass bottles) distributed to them at the prior week's clinic visit back to the clinic each week. Bottles were weighed using a Mettler Toledo balance to the nearest gram and recorded. Participants, parents, and clinic workers were blinded to the juice assignment. This was done by using juices made to have the same color and viscosity and numbering the juice bottles with one of two random three-digit numbers.

The following occurred at the baseline and at Week 2, 4, 6, and 8 clinic visits. Skin carotenoid status was measured by a trained research assistant using the BioPhotonic Scanner, a

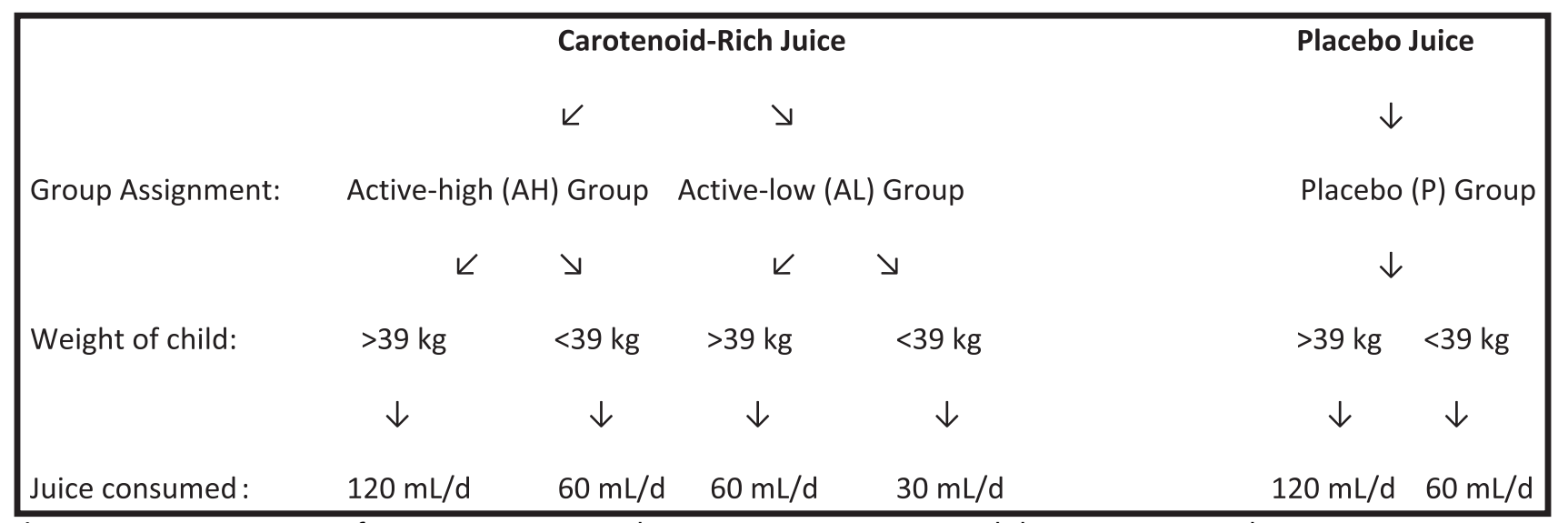

Figure 1. Juice assignments for 58 participants aged 5 to 17 years in a carotenoid dose-response study. 
portable RRS device. The child placed his or her palm (between the distal and proximal palmar region) against the light window of the scanner and held it there for 90 seconds. The scanner emitted a light and displayed a score in Raman intensity counts of 0 to $70,000+$. Each child was scanned twice using the same scanner and same palm at the clinic visits. In the case that there was $>2,000$ Raman intensity count difference between scanner scores, participants were scanned a third time. This was done to minimize the individual variation in scanner score. The two scanner scores that were within 2,000 Raman intensity counts were averaged. During the warm-up process, a black calibration cap was placed over the light window and the scanners self-calibrated using a patented process. Children and parents were blinded to the results of the scanner score.

At each clinic visit, participants completed a health checklist that reported lifestyle questions regarding the prior 7 days, including smoke exposure, nutritional supplement use, illness since prior clinic visit, and daily sun exposure. Parents assisted participants younger than age 10 years to complete these forms. Usual dietary intake was assessed from a total of three ( 2 weekend days and 1 weekday at least 7 days apart) computer-assisted 24-hour dietary recalls (24HDRs) using an automated self-administered 24-hour recall. In addition, participants completed weekly a 27 -item food frequency questionnaire (FFQ) that assessed $\mathrm{F} / \mathrm{V}$ intake during the previous week. Parents assisted participants younger than age 10 years to complete these forms. The participants were encouraged to maintain their usual diet throughout the duration of the study.

\section{Dietary Assessment}

The FFQ was a modified version of The Beverage and Snack Questionnaire (BSQ) developed and validated by Neuhouser and colleagues. ${ }^{20}$ The BSQ asks children to report how often they consumed items from a list of fruits, vegetables, snacks, and beverages during the past week. The BSQ was modified by adding additional questions about high-carotenoid-containing vegetables (HCV) and condensing snack items into more general categories, because they were not the focus of this study. This modified BSQ tool contained 27 items compared with the 19-item tool used by Neuhouser and colleagues. ${ }^{20}$

The tool used to collect the 24HDR was the National Cancer Institute ASA24-Kids-2012. ${ }^{21}$ This tool is based on the US Department of Agriculture Automated Multiple-Pass Method system involving five requests for intake data across the same 24HDR, which has been validated in children aged 10 years and older. ${ }^{22-24}$ Participants aged 10 years and older and parents of younger children completed the first recall during a clinic visit. Subsequent recalls were completed at home and the completion verified during clinic visits.

\section{Juice}

The carotenoid-rich juice was a product marketed under the brand name g3 Juice. Available to the public since 2005, it is currently sold in more than 40 markets around the world (including the United States). It is composed of four main fruit juices from concentrate: Gac (Mormordica cochinchinensis), Cili (rosa roxburghii tratt), Siberian pineapple (Hippophae rhamnoids, also known as sea buckthorn), and Chinese lycium (Lycium barbarum, also known as wolfberry) in addition to pear concentrate, grape concentrate, apple concentrate, acerola, natural flavor, citric acid, sodium benzoate (for stability), ascorbic acid, xanthan gum, and pectin. g3 Juice is composed of food-grade ingredients commonly found in the global food supply making it a safe, welltolerated juice product. It contains $20 \mathrm{kcal} / \mathrm{oz}, 5 \mathrm{mg}$ sodium, $5 \mathrm{~g}$ carbohydrate, $3 \%$ Daily Value of vitamin A, and $50 \%$ of the Daily Value of vitamin C.

The placebo juice was composed of apple juice concentrate, red grape juice concentrate, white grape juice concentrate, pear juice concentrate, pear purée, and Cili (rosa roxburghii tratt) as well as natural flavor, colorants (yellow \#6, red \#40, caramel color, and titanium dioxide), clouding agent (WILD Flavors and Specialty Ingredients Inc), xanthan gum, pectin, citric acid, and sodium benzoate (for stability). The placebo juice was made specifically for this study to have the same color and texture as the g3 Juice and contained undetectable amounts of carotenoids.

\section{Resonance Raman Spectroscopy}

Skin carotenoid status was determined by calculating the average height of the peak Raman absorbance signal obtained and quantified from excitation of skin carotenoids using a low-intensity blue $(\lambda=473 \mathrm{~nm})$ light-emitting diode light with green light $(510 \mathrm{~nm})$ detection $^{4}$ with the Biophotonic Scanner skin carotenoids reported as Raman intensity counts. The higher the count, the higher the concentration of carotenoid molecules detected at the site of measurement. The scanner reports total carotenoid value, not individual carotenoid counts, because there is overlap on the absorption spectra of each carotenoid.

\section{Statistical Analysis}

Statistics, including $t$ tests and analysis of variance (ANOVA), were used to examine differences, to describe the distribution of assessments and characteristics of the study population, and to compare means across subgroups (age, sex, ethnicity, BMI-for-age percentiles, weight, and juice groups). Association of potential confounding factors were examined, including smoke exposure, reported illness, supplement use, and sun exposure compared with skin carotenoid status at each of the data collection points. Linearity was tested by visual inspection of the scatter plots. To determine the normality of the data, Levene's statistics were conducted for all descriptive statistic calculations. For the linear regression analysis, skewness and kurtosis statistics were confirmed to be $\leq \pm 1$. Outliers within independent variables were topcoded to the next closest value. This was done by converting the scores to $Z$ values. ${ }^{25} Z$ values that were above the range of 1.96 , or 3 standard deviations, were changed to be the next value closest to 1.96 . This was done to be able to retain the outliers in the data set. Two to four data points were top-coded for the following independent variables: total F/V from baseline FFQ and averaged 24HDR; total HCV from baseline FFQ and averaged 24HDR; averaged beta carotene, alpha carotene, lycopene, lutein+zeazanthin, and total carotenoids from 24HDR.

Intraclass correlation coefficients (ICCs) were used to assess how well the scanner scores were correlated over multiple scannings obtained at one visit. In addition, ICC was used to assess assigned compared with actual juice consumption. 
Repeated measures ANOVA was used to assess the group differences in skin carotenoid status change. Because weight was more strongly correlated with scanner score than BMI and given that participants in the juice groups were assigned different amounts of juice based on their weight, weight was included as a covariate in the equation. Statistics were conducted using SPSS software version 22.0 (2013, IBM-SPSS Inc) with a $P$ value of 0.05 or below considered statistically significant.

\section{RESULTS}

Of 67 children who started the study, nine did not complete the study (13\%). Three were unable to attend clinic visits (two $\mathrm{AH}$ and one $\mathrm{P}$ ) and three did not care for the taste of the carotenoid-rich juice (two $\mathrm{AH}$ and one $\mathrm{AL}$ ). Three participants (one $\mathrm{AL}$, one $\mathrm{AH}$, and one $\mathrm{P}$ ) did not complete all of the study requirements. In the AL group, 20 participants were assigned and 18 completed the study. In the $\mathrm{AH}$ group, 23 participants were assigned and 19 completed the study. In the placebo group, 24 participants were assigned and 21 completed the study. The mean age for participants was $10.8 \pm 3.6$ years.

\section{Tests of Internal Validity}

We conducted a number of tests to ensure that the study's internal validity was sound. There were no differences in mean skin carotenoid status by sex, age groups, BMI, or juice groups at baseline (see Table 1). Children in the highest weight group had lower mean Raman intensity counts than

Table 1. Resonance Raman spectroscopy (RRS), serum carotenoid (SC), fruit and vegetable (F/V), and high-carotenoid vegetable

$(\mathrm{HCV})$ intake, by baseline characteristics of the study population $(n=58)$

\begin{tabular}{llllll} 
& RRS counts & & & & \\
& (Raman intensity & $24 \mathrm{HDR}^{\mathrm{a}}$ & $24 \mathrm{HDR}$ & Baseline FFQ $^{\mathrm{b}}$ & Baseline FFQ $^{\mathrm{n}(\%)}$ \\
counts) & $\mathrm{F} / \mathrm{V} \mathrm{(c)}$ & $\mathrm{HCV} \mathrm{(c)}$ & $\mathrm{F} / \mathrm{V}$ (servings) & $\mathrm{HCV} \mathrm{(servings)}^{\mathrm{c}}$ \\
\hline
\end{tabular}

\begin{tabular}{|c|c|c|c|c|c|c|}
\hline \multicolumn{7}{|l|}{ Grade/age (y) } \\
\hline Kindergarten-5th/5-10 & $28(48)$ & $23,919 \pm 862$ & $1.4 \pm 0.14$ & $0.26 \pm 0.04^{* * *}$ & $2.7 \pm 0.29$ & $0.77 \pm 0.09$ \\
\hline 6th-8th/11-13 & $18(31)$ & $22,696 \pm 941$ & $1.5 \pm 0.19$ & $0.29 \pm 0.06$ & $2.7 \pm 0.29$ & $0.82 \pm 0.12$ \\
\hline 9th-12th/14-17 & $12(21)$ & $21,877 \pm 1,628$ & $1.8 \pm 0.31$ & $0.61 \pm 0.10^{* * *}$ & $3.2 \pm 0.36$ & $1.06 \pm 0.18$ \\
\hline \multicolumn{7}{|l|}{ Sex } \\
\hline Male & $27(47)$ & $22,698 \pm 965$ & $1.6 \pm 0.17$ & $0.38 \pm 0.06$ & $2.7 \pm 0.28$ & $0.82 \pm 0.10$ \\
\hline Female & $31(53)$ & $23,499 \pm 766$ & $1.5 \pm 0.14$ & $0.31 \pm 0.04$ & $2.8 \pm 0.24$ & $0.87 \pm 0.09$ \\
\hline \multicolumn{7}{|l|}{ Race/ethnicity } \\
\hline White & $46(80)$ & $23,708 \pm 675$ & $1.6 \pm 0.16$ & $0.36 \pm 0.04$ & $2.9 \pm 0.20$ & $0.87 \pm 0.08$ \\
\hline Hispanic & $10(17)$ & $20,148 \pm 1,005$ & $1.4 \pm 0.32$ & $0.26 \pm 0.09$ & $2.5 \pm 0.51$ & $0.76 \pm 0.15$ \\
\hline Asian & $1(1.5)$ & 29,953 & 0.39 & 0.06 & 1.9 & 0.28 \\
\hline Polynesian & $1(1.5)$ & 17,983 & 3.2 & 0.68 & 2.7 & 1.29 \\
\hline \multicolumn{7}{|c|}{ Body mass index percentile } \\
\hline$>85$ th & $13(22)$ & $19,890 \pm 1,201$ & $1.4 \pm 0.29$ & $0.33 \pm 0.08$ & $2.7 \pm 0.37$ & $1.03 \pm 0.17$ \\
\hline$<5$ th & $4(7)$ & $23,423 \pm 3,304$ & $1.6 \pm 0.53$ & $0.41 \pm 0.22$ & $3.3 \pm 1.2$ & $0.78 \pm 0.28$ \\
\hline 5 th-85th & $41(71)$ & $24,074 \pm 652$ & $1.5 \pm 0.12$ & $0.34 \pm 0.04$ & $2.7 \pm 0.21$ & $0.80 \pm 0.08$ \\
\hline \multicolumn{7}{|l|}{ Weight (kg) } \\
\hline $16.9-30.2$ & $20(34)$ & $25,331 \pm 4,361^{\dagger}$ & $1.4 \pm 0.17$ & $0.27 \pm 0.04^{* * *}$ & $2.8 \pm 0.36$ & $0.66 \pm 0.10$ \\
\hline $30.9-47.7$ & $19(33)$ & $22,999 \pm 4,307$ & $1.4 \pm 0.15$ & $0.23 \pm 0.06^{* * *}$ & $2.6 \pm 0.31$ & $0.92 \pm 0.11$ \\
\hline $48.5-102.3$ & $19(33)$ & $21,041 \pm 4,581^{\dagger}$ & $1.7 \pm 0.24$ & $0.52 \pm 0.07^{* * *}$ & $2.9 \pm 0.27$ & $0.96 \pm 0.13$ \\
\hline \multicolumn{7}{|l|}{ Juice } \\
\hline Active high & $19(33)$ & $22,220 \pm 1,175$ & $1.5 \pm 0.22$ & $0.32 \pm 0.05$ & $2.4 \pm 0.25$ & $0.77 \pm 0.11$ \\
\hline Active low & $18(31)$ & $22,777 \pm 1,066$ & $1.4 \pm 0.18$ & $0.35 \pm 0.07$ & $3.0 \pm 0.31$ & $0.95 \pm 0.14$ \\
\hline Placebo & $21(36)$ & $24,141 \pm 929$ & $1.6 \pm 0.18$ & $0.35 \pm 0.06$ & $2.9 \pm 0.35$ & $0.82 \pm 0.11$ \\
\hline
\end{tabular}


children in the lowest weight group ( $P=0.009)$. The oldest age group (14 to 17 years) had higher intake of HCV than the youngest age group ( 5 to 10 years) $(P=0.04)$. There were no differences in mean skin carotenoid status among children who reported never or ever being sick during the study, and no child reported having exposure to tobacco smoke (data not presented). The amount of sun exposure significantly correlated to baseline skin carotenoid status. Children who had 0 to 29 minutes of sun exposure at baseline had 5,855 Raman intensity counts and 5,155 Raman intensity counts higher on average than those who had 1 to 2 hours or $>2$ hours, respectively $(P=0.032$ and $P=0.046)$. Children who reported taking multivitamins at baseline $(\mathrm{n}=18)$ had Raman intensity counts 4,645 higher at baseline on average than those who did not report multivitamin use $(P<0.001)$. However, these differences were not significant at Week $2,4,6$, or 8. Thus, very few differences existed at baseline before randomization and most other differences attenuated over the study.

We also considered it important to check the effectiveness of the randomization process. One-way ANOVA revealed no differences for RRS scanner scores, weight, age, and intake of HCV across the experimental groups at baseline $(P$ values $=0.40$, $0.13,0.40$, and 0.59 , respectively). Further, $\mathrm{a} \chi^{2}$ test showed that the sex differences across the groups were not significant $(P=0.58)$. A second nonsignificant $\chi^{2}$ test showed that participants who took supplements were evenly distributed across the juice groups $(P=0.16)$. Additional $\chi^{2}$ analyses showed that no differences existed across the groups in terms of the children becoming sick during the study $(P=0.38)$ or sun exposure at baseline $(P=0.25)$. Consequently, following randomization, no group differences existed before the beginning of the treatment. Because of this, the only control covariate used in the repeated measures ANOVA was weight, given that participants in the juice groups were assigned different amounts of juice based on their weight.

Another important validity check was whether participants actually consumed the assigned amount of juice. Assigned compared with actual juice consumption, measured through weighing the juice bottles on a weekly basis, were highly correlated (ICC $=0.93 ; P<0.001$ ). This suggests that participants' compliance was quite high.

We also examined our measures of diet as well as changes in diet as an internal validity check. A repeated-measures ANOVA revealed that participants remained consistent in their consumption of $\mathrm{F} / \mathrm{V}$ and $\mathrm{HCV}$ throughout the study based on the FFQ ( $P=0.34$ and $P=0.17$, respectively). In addition, there were no significant differences in consumption of $F / V$ or HCV changes between the three juice groups $(P=0.28$ and $P=0.34$ ). The results of multivariable linear regression models that controlled for the child's weight, found that selfreported F/V and HCV consumption using FFQ and 24HDR correlated significantly with skin carotenoid status. The results are listed in Table 2. Finally, the duplicate or triplicate (if scores differed by $>2,000$ units) RRS intensity counts from each clinic visit were also highly correlated (baseline ICC $=0.93$ $[P<0.001]$, Week 2 ICC $=0.8[P<0.001]$, Week 4 ICC $=0.99$ $[P<0.001]$, Week 6 ICC $=0.93[P<0.001]$, and Week 8 ICC $=0.94$ $[P<0.001])$, and so the two RRS intensity counts that were within 2,000 units were averaged. This suggests that the scanners used to gather the RRS scores were reliable over time.
Table 2. Multivariable linear regression analysis of the $R^{2}$ and $\beta$ representing the relationship between total carotenoids measured by baseline resonance Raman spectroscopy (RRS) and fruit and vegetable (F/V) and carotenoids intake estimated from baseline food frequency questionnaire (FFQ) and three averaged 24-hour dietary recalls (24HDRs)

\begin{tabular}{|c|c|c|c|c|}
\hline take measure & $\begin{array}{l}\text { RRS } \\
\text { model } \\
R^{2 \mathrm{a}}\end{array}$ & $\begin{array}{l}\text { RRS } \\
\beta \\
\end{array}$ & $\begin{array}{l}P \\
\text { value } \\
\end{array}$ & $\begin{array}{l}\text { Estimated } \\
\text { change in } \\
\text { Raman intensity } \\
\text { counts with } \\
\text { consumption } \\
\text { of } 1 \text { portion F/V }\end{array}$ \\
\hline $\mathrm{FQ} F / \mathrm{V}^{\mathrm{b}}$ & $0.22^{* *}$ & .30 & 0.001 & 1,009 \\
\hline$F Q \mathrm{HCV}^{\mathrm{C}}$ & $0.15^{*}$ & .16 & 0.011 & 1,384 \\
\hline $24 H D R ~ F / N^{d}$ & $0.16^{* *}$ & .20 & 0.007 & 1,062 \\
\hline 24HDR HCV & $0.16^{* *}$ & .21 & 0.007 & 3,436 \\
\hline 24HDR TC & $0.17^{* *}$ & .23 & 0.005 & 241 \\
\hline 24HDR lycopene ${ }^{g}$ & $0.16^{* *}$ & .19 & 0.008 & 256 \\
\hline $\begin{array}{l}\text { 24HDR alpha } \\
\text { carotene }^{\text {h }}\end{array}$ & $0.14^{*}$ & .45 & 0.013 & 2,489 \\
\hline $\begin{array}{l}\text { 24HDR beta } \\
\text { carotene }^{i}\end{array}$ & $0.14^{* *}$ & .13 & 0.014 & 555 \\
\hline 24HDR LZ & $0.13^{* *}$ & .074 & 0.021 & 717 \\
\hline \multicolumn{5}{|c|}{ 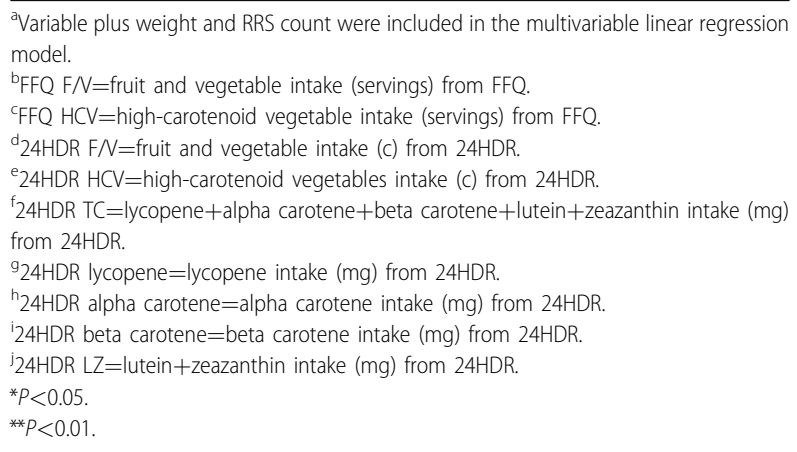 } \\
\hline
\end{tabular}

\section{Effect of Juice Consumption}

By Week 2, there were significant differences in skin carotenoid status in the carotenoid-rich juice groups (both $\mathrm{AH}$ $[P=0.002]$ and $A L[P=0.02]$ but not in $P[P=0.61])$. The repeated-measures ANOVA revealed a main effect for time $(P<0.001)$, juice group $(P=0.004)$, and weight $(P=0.03)$. The time $\times$ juice group interaction was also significant $(P<0.001)$, although the time $\times$ weight interaction was not $(P=0.53)$. Figure 2 shows the graph of the interaction using estimated marginal means. It shows that after accounting for weight of the participants, the AH and AL groups had estimated mean increases in skin carotenoid status of 11,515 and 10,009 Raman intensity counts, respectively. These changes were significantly different $(P<0.001)$. The estimated change in the $P$ group (985 Raman intensity counts) was not statistically significant. There was no significant difference in change between the two experimental groups. The results of a 


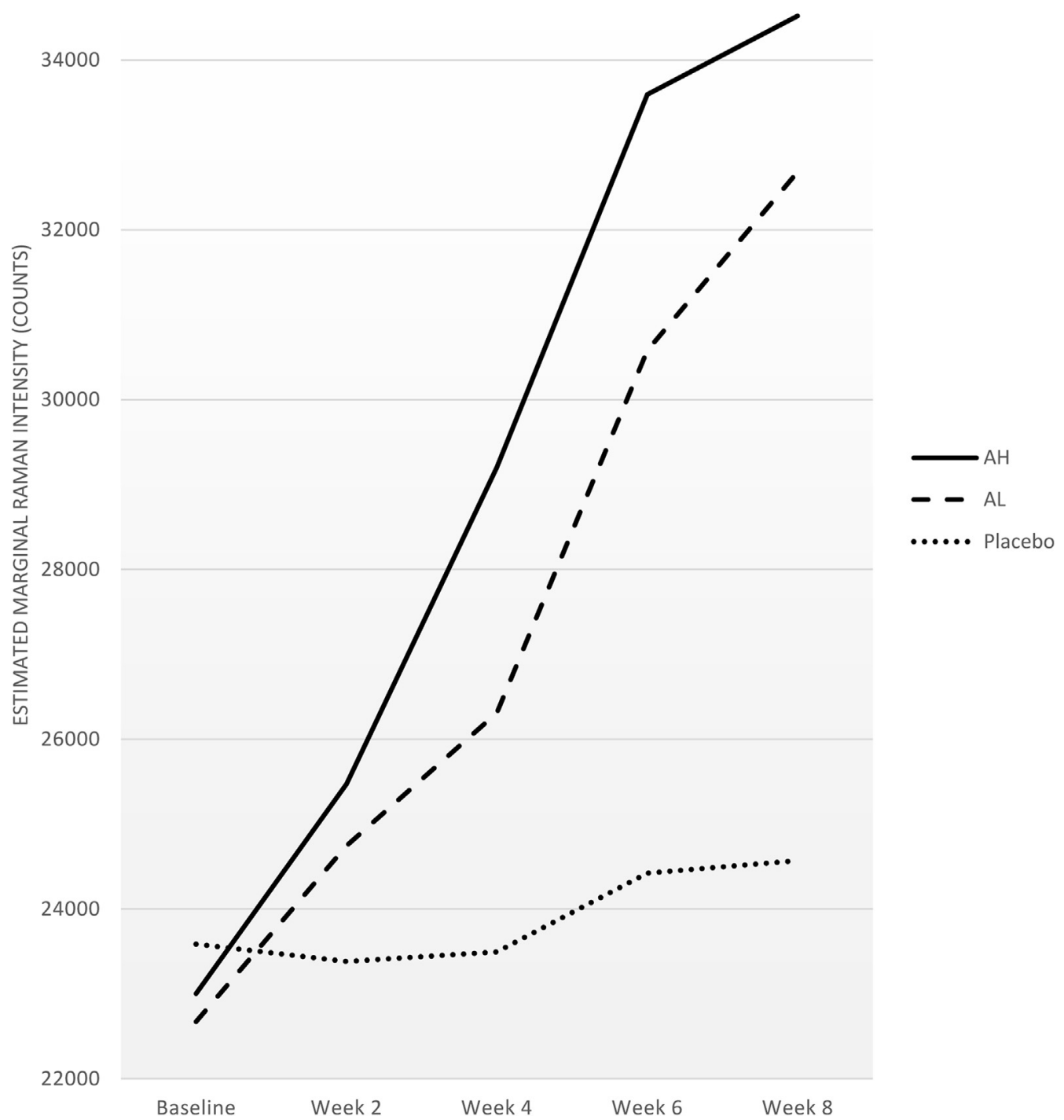

Figure 2. Change in skin carotenoid status over 8 weeks among children aged 5 to 17 years consuming 30 to $60 \mathrm{~mL}$ ( $\mathrm{AL}$ ) or 60 to $120 \mathrm{~mL}(\mathrm{AH})$ carotenoid-rich juice or placebo using resonance Raman spectroscopy and measured in Raman intensity counts.

multivariable linear regression model, which controlled for child's weight, found that consuming $30 \mathrm{~mL}$ juice $(2.75 \mathrm{mg}$ carotenoid) raised skin carotenoid status by 56 Raman intensity counts on average $(P<0.001)$.

\section{DISCUSSION}

In this study, consumption of a known dose of carotenoids ( 2.75 to $11 \mathrm{mg}$ ) from a carotenoid-rich juice $(2.75 \mathrm{mg} / 30 \mathrm{~mL})$ significantly increased skin carotenoid status over an 8-week period among children aged 5 to 17 years. Similarly, a study of children who received supplemented rice with gac fruit (3.5 $\mathrm{mg}$ carotenoids) or beta carotene powder $(5.0 \mathrm{mg}$ carotenoids) compared with control diet across 30 days had significantly higher plasma beta carotene concentrations. ${ }^{15}$
No statistically significant difference in skin carotenoid status between participants consuming the carotenoid-rich high-dose and carotenoid-rich low-dose juice was measured during the study. This is important because consuming the amount of carotenoids found in the lower carotenoid-rich dose of juice represents an amount that would be a more realistic addition to a child's diet. The amount of carotenoids found in the lowest carotenoid-rich juice dose is equivalent to the amount found in approximately 23 to $46 \mathrm{~g}$ cooked carrots (approximately 2.75 to 5.5 mg carotenoids). ${ }^{26}$ These results are similar to what Tanumihardjo and colleagues ${ }^{27}$ found when feeding a high (11 mg carotenoids), low (4.4 mg carotenoids), and no-carotenoid muffin to 10 children. Participant serum carotenoid levels increased significantly with no significant differences in high 
consumers and low consumers of carotenoid muffins. ${ }^{27}$ The reason for this large individual variation in serum carotenoid is unclear in the literature and has been seen in highcarotenoid food and supplement studies. ${ }^{27-29}$ It has been hypothesized to be due to differences in absorption, altered metabolism, or differences in the ability to cleave beta carotene to vitamin A. $^{28}$

Significant correlations between skin carotenoids and total F/V and HCV from FFQ and 24HDR were observed. These results are comparable to other published works and are described in a previous publication. ${ }^{7,8,30,31}$ In this study, the strongest self-reported diet correlation to skin carotenoid status was F/V intake report using FFQ although the estimated change in Raman intensity counts with consumption of one portion of $\mathrm{F} / \mathrm{V}$ was similar for 24HDR F/V (1,009 vs 1,062 Raman intensity counts, respectively). This is similar to the findings in a previous study. ${ }^{8}$ Using the FFQ method for diet recall is faster for participants to complete which is beneficial, especially when conducting studies in a school setting. Based on these results, FFQ appears to be an adequate method to assess $\mathrm{F} / \mathrm{V}$ intake in children.

Published studies have found variation in the effects of high-carotenoid fruits and vegetables on serum carotenoid levels. ${ }^{15,16,32}$ De Pee and colleagues ${ }^{32}$ found that orange fruit was found to be more effective than dark-green, leafy vegetables (with a large range of apparent effectiveness) in increasing serum concentrations of beta carotene in schoolchildren in Indonesia. The carotenoid concentration of the carotenoid-rich juice in the current study is known, but the bioavailability of carotenoids is not known. Vuong and colleagues ${ }^{15}$ found that the beta carotene from gac fruit, a component of the carotenoid-rich juice used in our study, is a good source of provitamin A carotenoids in children.

No significant mean differences were found in skin carotenoid levels between sexes, BMI, or illness. Similar results were found in a previous study in children ${ }^{8}$ and could be due to several factors. For example, few children were in the high or low BMI groups and the severity of illness was not quantified. Other studies in children comparing $\mathrm{F} / \mathrm{V}$ intake to serum carotenoid levels ${ }^{8,20,32}$ and skin carotenoid levels ${ }^{21}$ found significant associations after adjusting for BMI. However, weight was a stronger positive predictor in this study, possibly because it better reflects the volume of distribution of a given amount of carotenoid intake.

This study had several strengths and limitations. To our knowledge, this is the first study that looked at the effect of feeding children a carotenoid-rich beverage and observing the change in skin carotenoid status over an 8-week period of time. The diet intervention was a commercially available juice that provided consistent, measurable amounts of dietary carotenoids. Because the placebo juice was consistent in color and thickness, the participants were blinded to their assigned juice. The study took place during winter months so sun exposure was limited, providing a natural control for this important confounder to relationships between dietary intake of carotenoids and skin carotenoid concentrations.

The limitations of this study include the geographically limited area in which the study took place, so it may not be generalizable to other populations of children that include more diversity in skin color and other factors. Participants were intentionally selected to represent the race/ethnicity of the school-age population in the region, which consists of
$<1 \%$ African Americans. Participants who consumed carotenoid-containing multivitamin/mineral supplements were not excluded from the study and had a higher skin carotenoid status at baseline; however, there were no differences in the proportion of participants who consumed multivitamin/mineral supplements between participants randomized to the three experimental conditions. By Week 2, the differences disappeared. In addition, participants who spent more time outdoors had significantly lower skin carotenoid status but were not excluded. This was potentially due to the oxidation effects of poor air quality during winter months in the region, which has been shown to lower serum carotenoid levels. ${ }^{33}$ Again, that effect disappeared by the second week of the study. Skin carotenoid status was not compared with serum levels in this study. However, previous work has found that these levels are highly correlated in both adults $^{7}$ and children ${ }^{8}(r=0.81$ and $r=0.79$, respectively, both $P$ values $<0.001$ ).

\section{CONCLUSIONS}

Skin carotenoid status, as measured by the RRS, is sensitive to consistent modest change in carotenoid intake among children. The level of increase in carotenoid intake provided by the lowest intake of carotenoid-rich juice $(30$ to $60 \mathrm{~mL}$ ) is similar to the amount of carotenoids found in approximately 23 to $46 \mathrm{~g}$ cooked carrots, which appears to be a reasonable increase in intake in the diet of a child. ${ }^{34}$ Thus, skin carotenoid status may be used as a biomarker of change in carotenoid intake in children. This biomarker could be valuable in a clinical setting to measure dietary change in clients engaging in nutrition counseling.

\section{References}

1. Esfahani A, Wong JM, Truan J, et al. Health effects of mixed fruit and vegetable concentrates: A systematic review of the clinical interventions. J Am Coll Nutr. 2011;30(5):285-294.

2. Crichton GE, Bryan J, Murphy KJ. Dietary antioxidants, cognitive function and dementia-A systematic review. Plant Foods Hum Nutr. 2013;68(3):279-292.

3. Deming DM, Boileau TW-M, Heintz KH, Atkinson CA, Erdman JW Carotenoids: Linking chemistry, absorption, and metabolism to potential roles in human health and disease. In: Cadenas E, Packer L, eds. Handbook of Antioxidants. New York, NY: Marcel-Dekker; 2002: 189-221.

4. Smidt CR, Burke DS. Nutritional significance and measurement of carotenoids. Curr Top Nutraceutical Res. 2004;2:79-91.

5. Institute of Medicine, Food and Nutrition Board. Dietary Reference Intakes for Vitamin C, Vitamin E, Selenium, and Carotenoids. Washington, DC: The National Academies Press; 2000.

6. Mayne ST, Cartmel B, Scarmo S, Jahns L, Ermakov IV, Gellermann W Resonance Raman spectroscopic evaluation of skin carotenoids as a biomarker of carotenoid status for human studies. Arch Biochem Biophys. 2013;539(2):163-170.

7. Zidichouski JA, Mastaloudis A, Poole SJ, Reading JC, Smidt CR. Clinical validation of a noninvasive Raman spectroscopic method to assess carotenoid nutritional status in humans. J Am Coll Nutr. 2009;28(6): 687-693.

8. Aguilar SS, Wengreen HJ, Lefevre M, Madden GJ, Gast J. Skin carotenoids: A biomarker of fruit and vegetable intake in children. J Acad Nutr Diet. 2014;114(8):1174-1180.

9. Ermakov IV, Sharifzadeh M, Ermakova M, Gellermann W. Resonance Raman detection of carotenoid antioxidants in living human tissue. J Biomed Opt. 2005;10(6):064028.

10. Burrows TL, Warren JM, Colyvas K, Garg ML, Collins CE. Validation of overweight children's fruit and vegetable intake using plasma carotenoids. Obesity (Silver Spring). 2009;17(1):162-168. 
11. Rerksuppaphol S, Rerksuppaphol L. Effect of fruit and vegetable intake on skin carotenoid detected by non-invasive Raman spectroscopy. J Med Assoc Thai. 2006;89(8):1206-1212.

12. Natarajan L, Flatt SW, Sun X, et al. Validity and systematic error in measuring carotenoid consumption with dietary self-report instruments. Am J Epidemiol. 2006:15;163(8):770-778.

13. Scarmo S, Henebery K, Peracchio H, Cartmel B, Lin H, Ermakov IV. Skin carotenoid status measured by resonance Raman spectroscopy as a biomarker of fruit and vegetable intake in preschool children. Eur J Clin Nutr. 2012;66(5):555-560.

14. Tang G, Hu Y, Yin SA, et al. $\beta$-Carotene in golden rice is as good as $\beta$ carotene in oil at providing vitamin A to children. Am J Clin Nutr 2012 Sep;96(3):658-664.

15. Vuong le T, Dueker SR, Murphy SP. Plasma beta-carotene and retinol concentrations of children increase after a $30-d$ supplementation with the fruit Momordica cochinchinensis (gac). Am J Clin Nutr. 2002;75(5):872-879.

16. Nawiri MP, Nyambaka H, Murungi JI. Sun-dried cowpeas and amaranth leaves recipe improves $\beta$-carotene and retinol levels in serum and hemoglobin concentration among preschool children. Eur J Nutr. 2013;52(2):583-589.

17. Persson V, Ahmed F, Gebre-Medhin M, Greiner T. Increase in serum beta-carotene following dark green leafy vegetable supplementation in Mebendazole-treated school children in Bangladesh. Eur J Clin Nutr. 2001;55(1):1-9.

18. Jahns L, Johnson LK, Mayne ST, et al. Skin and plasma carotenoid response to a provided intervention diet high in vegetables and fruit: Uptake and depletion kinetics. Am J Clin Nutr. 2014;100(3): 930-937.

19. Le Marchand L, Hankin JH, Carter FS, et al. A pilot study on the use of plasma carotenoids and ascorbic acid as markers of compliance to a high fruit and vegetable dietary intervention. Cancer Epidemiol Biomarkers Prev. 1994;3(3):245-251.

20. Neuhouser ML, Lilley S, Lund A, Johnson DB. Development and validation of a beverage and snack questionnaire for use in evaluation of school nutrition policies. J Am Diet Assoc. 2009;109(9):15871592.

21. National Cancer Institute, National Institutes of Health. ASA24TMKids Web site. http://riskfactor.cancer.gov/tools/instruments/asa24] researcher/. Published September 2012. Accessed January 2013.

22. Baranowski T, Islam N, Baranowski J, et al. The food intake recording software system is valid among fourth-grade children. J Am Diet Assoc. 2002; 102(3):380-385.
23. Baranowski T, Islam N, Baranowski J, et al. Comparison of a Webbased versus traditional diet recall among children. J Acad Nutr Diet. 2012;112(4):527-532.

24. Subar AF, Kirkpatrick SI, Mittl B, et al. The Automated SelfAdministered 24-hour dietary recall (ASA24): A resource for researchers, clinicians, and educators from the National Cancer Institute. J Acad Nutr Diet. 2012;112(8):1134-1137.

25. Lipsey M, Wilson D. Practical Meta-Analysis. Thousand Oaks, CA Sage; 2001.

26. Holden JM, Eldridge AL, Beecher GR, et al. Carotenoid content of U.S. foods: An update of the database. J Food Comp Anal. 1999;12:169-196.

27. Tanumihardjo SA, Horvitz MA, Dosti MP, Simon PW. Serum alphaand beta-carotene concentrations qualitatively respond to sustained carrot feeding. Exp Biol Med (Maywood). 2009;234(11): 1280-1286.

28. Borel P, Grolier P, Mekki N, et al. Low and high responders to pharmacological doses of beta-carotene: Proportion in the population, mechanisms involved and consequences of beta-carotene metabolism. J Lipid Res. 1998;39(11):2250-2260.

29. Crane TE, Kubota C, West JL, Kroggel MA, Wertheim BC, Thomson CA. Increasing the vegetable intake dose is associated with a rise in plasma carotenoids without modifying oxidative stress or inflammation in overweight or obese postmenopausal women. J Nutr. 2011;141(10):1827-1833.

30. Resnicow K, Odom E, Wang T, et al. Validation of three food frequency questionnaires and 24-hour recalls with serum carotenoid levels in a sample of African-American adults. Am J Epidemiol. 2000;152(11):1072-1080.

31. Neuhouser ML, Rock CL, Eldridge AL, et al. Serum concentrations of retinol, alpha-tocopherol and the carotenoids are influenced by diet, race and obesity in a sample of healthy adolescents. J Nutr. 2001;131(8):2184-2191.

32. de Pee S, West CE, Permaesih D, Martuti S, Muhilal, Hautvast JG Orange fruit is more effective than are dark-green, leafy vegetables in increasing serum concentrations of retinol and beta-carotene in schoolchildren in Indonesia. Am J Clin Nutr. 1998;68(5): 1058-1067.

33. Romieu I, Castro-Giner F, Kunzli N, Sunyer J. Air pollution, oxidative stress and dietary supplementation: A review. Eur Respir J. 2008:31 (1):179-197.

34. Knai C, Pomerleau J, Lock K, McKee M. Getting children to eat more fruit and vegetables: A systematic review. Prev Med. 2006;42(2) 85-95.

\section{AUTHOR INFORMATION}

S. S. Aguilar is a senior research dietitian, Center for Human Nutrition Studies, H. J. Wengreen is an associate professor, Nutrition, Dietetics, and Food Sciences, and J. Dew is an associate professor, Family, Child, and Human Development, all at Utah State University, Logan.

Address correspondence to: Sheryl S. Aguilar, MS, RD, Center for Human Nutrition Studies, Utah State University, 9815 Old Main Hill, Logan, UT 84322-981. E-mail: Sheryl.aguilar@usu.edu

STATEMENT OF POTENTIAL CONFLICT OF INTEREST

NuSkin, LLC, provided the active and placebo juice for the study. After the conclusion of this study, S. S. Aguilar was a study coordinator for an unrelated study funded by NuSkin.

\section{FUNDING/SUPPORT}

This study was funded through the Center for Human Nutrition Studies at Utah State University.

\section{ACKNOWLEDGEMENTS}

The authors thank the participants, the nutrition and dietetics students who assisted in data collection, and the staff of the Center for Human Nutrition Studies at Utah State University. 